\title{
Assessment of Dual-frequency Signal Quality Monitor to Support CAT II/III GBAS
}

\author{
Junesol Song, Carl Milner, Ikhlas Selmi, Sara Bouterfas, ENAC, Université de Toulouse, France \\ Olivier Julien, $u$-Blox AG, Switzerland
}

\section{BIOGRAPHY (IES)}

Junesol Song is a postdoctoral fellow in Telecom laboratory at the Ecole Nationale de l'Aviation Civile (ENAC) in Toulouse, France. She received B.S. and Ph.D. degrees in aerospace engineering from Seoul National University, South Korea. Her research interests include dual-frequency/multi-constellation GBAS fault monitoring, multi-GNSS Network RTK, Compact RTK, carrier phase-based algorithms and positioning, and remote sensing.

Dr. Carl Milner is an assistant professor within the Telecom Lab at the Ecole Nationale de l'Aviation Civile, Toulouse France. He currently lectures on many aspects of navigation science and technology including radio navigation aids, signal processing, positioning algorithms and GNSS for civil aviation. His research work addresses the design of integrity monitoring algorithms for current GNSS signals and the future multi-constellation multifrequency environment.

Dr. Ikhlas Selmi is currently part of the SIGnal processing and NAVigation (SIGNAV) research group of Telecom lab at ENAC (Ecole Nationale de l'Aviation Civile). She received a Master of Science in Telecommunication and Signal Processing from ESIEE (Ecole Supérieure d'ingénieur en Electronique et Electrothechnique) and a Master of Research in High Frequency Communication System from University Paris-Est, Marne la Vallée. She obtained her Ph.D. in 2013 from Telecom SudParis. Her Ph.D thesis dealt with optimizing indoor positioning system based on GNSS transmitters, and more specifically, on mitigating interference between indoor and outdoor signals and simplifying the system's architecture. Then she was a post-doctoral fellow at University of LittoralCôte-d'Opale (ULCO) where she worked on designing an underwater positioning system for low depth environment. She is currently working on GNSS Signal distortion generated by the satellite payload.

Sara Bouterfas is a research assistant at the Signal Processing and Navigation (SIGNAV) research group of the TELECOM laboratory of ENAC, in Toulouse, France. She received her Engineering degree (equivalent MSc.) in Information Technology and Air Traffic Management from ENAC. Her research interests include multi-constellation GBAS/SBAS fault monitoring and spoofing.

Dr. Olivier Julien is a Senior Principal Engineer in u-blox AG, Switzerland since December 2018. He was the head of the Signal Processing and Navigation (SIGNAV) research group of the TELECOM laboratory of ENAC, in Toulouse, France. He received his engineer degree in 2001 in digital communications from ENAC and his PhD in 2005 from the Department of Geomatics Engineering of the University of Calgary, Canada. His research interests are turned towards the use of satellite-based navigation systems for safe navigation.

\footnotetext{
ABSTRACT

This paper assesses the performance of the signal quality monitors for the Ground-Based Augmentation System (GBAS) which supports Category (CAT) II and III precision approach. Three types of monitors are used for signal deformation faults: Honeywell signal quality monitor (SQM), ENAC Code-Carrier Divergence (CCD) monitor and a proposed Divergence-Free (DF)-Innovation monitor. The existing Honeywell SQM and ENAC CCD monitors have some response time due to the smoothing filter used for their metrics. Consequently, the performance of those monitors is limited right after the fault onset. To improve the monitor performance in this time period, the DF-Innovation monitor has been proposed. The performance of the monitors has been assessed by comparing the minimum value of the probabilities of missed detection of three monitors and the required probability of missed detection according to the differential range error, which is defined in the standard document. As a result, for GBAS Approach Service Types
} 
(GAST) F, the probability of missed detection of the monitor was compliant with respect to the requirements for all fault cases and receiver configurations, for GPS L1/L5 and Galileo E1/E5. In addition, we observed that the proposed DF-Innovation monitor is effective in reducing time delay, which is the required time at airborne from filter initialization time to the time when the airborne user incorporates the measurement and correction for navigation. To be more specific, the use of the proposed monitor can reduce the time delay by $80 \%$ compared to the case without using the proposed monitor, and moreover, it can even reduce the value of time delay below 50 second, which is the recommended value currently.

\section{INTRODUCTION}

Signal Quality Monitoring (SQM) must detect one of the ranging source faults, satellite signal distortions to prevent user receivers from misleading information (MI) in high-integrity augmentation systems such as the Ground-Based Augmentation System (GBAS) and the Satellite-Based Augmentation System (SBAS). These distortions have occurred on the GPS C/A code due to a satellite malfunction [1] and could potentially reoccur in the future in spite of improved satellite design. Consequently, under failure the correlation function is distorted and it leads to an incorrectly estimated code delay, leading to a bias-like error being included in the pseudorange measurement. Moreover, this range error can vary according to receiver tracking configurations in terms of chip spacing, pre-correlation filter type and its bandwidth. To protect ground and user receivers against this type of range fault, it is mandatory to have monitors to detect and remove those distorted signals.

Currently, single-frequency GBAS solutions known as GBAS Approach Service Types (GAST) C and D can support CAT I and CAT II/III precision approach respectively using the GPS L1 C/A signal. In the future, GBAS will utilize additional modernized signals such as GPS L5 and Galileo E1c/E5a to improve navigation accuracy and integrity performance under a solution designated GAST F to support CAT II/III. The additional signals should also be protected against signal distortions. GAST D provides requirements of the Probability of Missed Detection (PMD) for monitors to detect ranging source faults [2] and the same requirement may be applied to GAST F subject to caveats regarding the processing mode. An ionosphere-free processing leads to greater differential ranging errors under failure and this would need to be taken in to account. The PMD compliance of signal quality monitors for GAST D has been assessed for GPS L1 C/A [3], however, the assessment for GAST F using multi-constellation and multifrequency signals has not been conducted thoroughly.

This study focuses on the assessment of GBAS signal quality monitors under the proposed GAST F solution. The threat model for GPS L1 C/A signal has been defined in the International Civil Aviation Organization (ICAO) standards [4]. Agreement on the threat space of the modernized signals has not yet been reached at standardization level. However, research has proposed threat spaces for those signals, taking into account different chipping rates and correlation functions [5]. Among them, the threat spaces for Galileo signals are under a validation process prior to standardization. Based on these threat models, signal quality monitors are tested for dual-frequency modes. To be more specific, dual-frequency signal quality monitors for GPS L1/L5 and Galileo E1c/E5a are tested under dual-frequency navigation mode (i.e. ionosphere-free (IF) navigation mode) whether they comply with the PMD requirement according to the differential range errors. The differential range error in IF navigation mode can have larger values than those in the single-frequency navigation mode, because the range errors on the two frequencies of L1/E1 and L5/E5a are multiplied by 2.26 and -1.26 , respectively, according to the factors used to form the linear combination of an IF measurement.

In the work undertaken, new dual-frequency monitors have been designed; as well as the definition of a combination of monitors to protect against signal distortions. Three types of monitors are used to detect signal distortions in this study: the first is the signal quality monitor developed by Honeywell in 2006 [6], the second is a variation of the Code-Carrier Divergence (CCD) monitor developed by ENAC [7] and the third is a novel Divergence-Free (DF) Innovation monitor. This novel DF-Innovation monitor compares a dummy pseudorange computed using the divergence-free combination to the predicted DF pseudorange at the current epoch, which is propagated from the previous epoch using variations of the carrier phase. Accordingly, the novel monitor is sensitive to capturing step faults in the pseudorange measurements, yet is not impacted by ionospheric noise which reduces the efficiency of existing step detectors. When determining thresholds for the monitors of the CCD and the DF-Innovation monitor, statistics were computed using measurements collected from the GBAS reference receivers at Tenerife airport.

In this assessment, not only the performance of the monitors but also the differential range errors between ground and airborne receivers play a critical role is determining PMD compliance. The differential range error can vary according to the bias errors associated with signal distortion as well as a function of the different pseudorange smoothing filter initialization times at ground and airborne sides. The nominal case can be that both filters at the ground and airborne are initialized far earlier than the fault onset time (post-convergence case). The worst case for the PMD compliance occurs when the airborne smoothing is initialized just after the 
fault onset time. In this case the differential range error becomes large before the fault is fully incorporated in the monitor (transient case). In this case the pseudorange smoothing filter at the ground is initialized far earlier which is guaranteed by ground subsystem wait times specified in the standards. Consequently, PMD compliance was tested for various filter initialization times of the airborne receiver.

The monitoring performance also varies with the time constant used for pseudorange smoothing filter. A 30-second time constant is used for GAST D solution. For GAST F, it is under discussion to use a 100-second time constant in order to leverage the existing 100s corrections provided in GBAS Message Type 1 (MT1). Thus, this study further assesses the effect of the time constant used for the pseudorange smoothing filter on the PMD compliance.

Simulations have then been performed to investigate the monitoring performance and check under what conditions compliance with the requirement is achieved. The simulation has the following procedure: firstly, the GNSS code sequences are generated and the distortion is added onto the code sequences according to values given by the threat model parameters, which represent the lead/lag of a chip, resonant frequency and damping factor. This is made for the full range of parameter combinations within the threat space. Secondly, the correlation function is computed, and it is applied to the pre-correlation filters according to the types of filters and associated bandwidths. By inspecting the filtered correlation function with the Early-Late correlator, the code tracking offset is computed. The resultant code delay can vary according to the values of chip spacings selected for Early-Late correlator. Thirdly, the range error can be computed by comparing code delay values from nominal and distorted correlation functions, and it represents the error associated with the signal distortion. The ground and airborne receivers experience different range errors if they have different receiver configurations. Finally, the computed range error is added to the simulated pseudorange measurement. The Honeywell signal quality monitor uses multi-correlator outputs directly to detect the signal distortion. On the other hand, the CCD and DF-Innovation monitor use the code pseudorange and phase measurements to detect the effect of signal distortion in the range domain. Taking into account the different ground latency for GAST D and proposed latency for GAST F, which reflects the update interval of the correction message and ground processing delay, the PMD is plotted against the absolute value of the differential range error. In this simulation, Time-To-Detect and Affect Broadcast (TTDABA) is set to 1.5 seconds for GAST F, since the update interval of the integrity message must remain the same as for GAST D [8]. As a result, the proposed dual-frequency signal quality monitoring solution within GAST F, which consists of the Honeywell signal quality monitor, ENAC DF CCD monitor and new DF-Innovation monitor complies with the PMD requirement for GPS L1/L5 and Galileo E1c/E1a signals when the 100-second time constant is used for pseudorange smoothing filter. The 145-second time delay, which indicates the time delay from initialization of the airborne filter to incorporation in the solution, needs to be set for PMD compliance under the transient case when only the existing Honeywell SQM and ENAC CCD monitors are used. On the other hand, when the proposed DF-Innovation monitor is used additionally, the time delay can be reduced to 30 seconds. In addition, from the results of an additional test for the pseudorange smoothing filter with the 30 -second time constant, it was observed that the effect of the smoothing filter time constant acts differently on post-convergent and transient cases. That is, a longer time constant gives more compliant results for the post-convergent case, on contrary, a shorter time constant is more beneficial for the transient case.

\section{MONITORS USED FOR SIGNAL DEFORMATION FAULTS}

There have been few related works undertaken on the assessment of the signal deformation monitors for GAST D and F. For GAST D, Honeywell has assessed the minimum value of the PMD of Honeywell SQM, CCD monitors and step detector for GPS L1 C/A signal [9]. It concludes that the under 25-sec of time delay, the PMD of the monitor was compliant for all fault cases and receiver configurations. However, the work has been done only for the GPS L1 C/A signal. For GAST F, Thales has assessed the PMD of three monitors for signal deformation faults for GPS and Galileo signals [10]. However, the monitors used for the test and the threat space for GPS L5 and Galileo signals were not presented within the public domain. In this paper, the performance of the existing Honeywell SQM, ENAC CCD and the proposed DF-Innovation monitors is assessed for GAST F using the standardized threat space for GPS L1 C/A and the previously proposed threat spaces for GPS L5 and Galileo E1c/E5a [5]. 
The Honeywell SQM monitor utilizes the correlator outputs from eight different correlator locations as shown in Error! Reference source not found. [6]. The differences of correlator outputs between neighboring correlator locations are computed to form a vector, $X$, which has seven elements. The mean of the vector $X$ is subtracted from the vector $X$ and it is further normalized. Using the
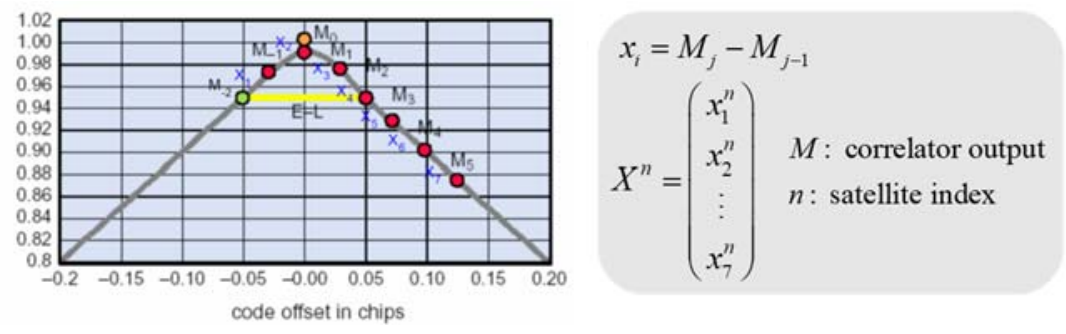

$$
\begin{aligned}
Y^{n}=F\left(X^{n}-\mu^{n}\right) \quad \mu: & \text { vector of mean value of } x \\
\text { where, } \quad F & =D^{-1 / 2} U^{-1} \\
P & =\operatorname{cov}\left(X^{n}\right)=U D U^{T} \\
\text { Metric: } T^{n}=\left(Y^{n}\right)^{T}\left(Y^{n}\right) & \sim \text { Chi-square distribution }
\end{aligned}
$$

Figure 1 Description of Honeywell SQM Monitor [5]

normalized vector, the square sum error, denoted as $T$, is computed and is used as the metric of the Honeywell SQM monitor. In this test, the theoretical standard deviations of correlator outputs are computed based on the approach introduced in [5]. These theoretical values have been validated by the actual correlator output collection at the ENAC site using an iFen SX3 software receiver. The Honeywell SQM metric follows a chi-square distribution with 7 degrees of freedom. The continuity requirement for signal deformation fault is $1.5 \times 10^{-7}$ per 15 seconds. Since a 30 -second time constant for a smoothing filter for the correlator outputs is used in the test, four independent samples are assumed in 15 seconds of exposure time following the updated analysis in [11]. Furthermore, considering a maximum of 15 tracked satellites, a threshold of 53.87 for the Honeywell SQM monitor is obtained.

ENAC DF CCD monitors [7] are also used in the assessment. These monitors are based on the rate of the DF measurement combinations, which can be defined as the following equations.

$$
\begin{aligned}
& \dot{\chi}_{D F 1}=\dot{\rho}_{1}-\dot{\phi}_{1}-\frac{2}{\gamma-1}\left(\dot{\phi}_{1}-\dot{\phi}_{5}\right)=\dot{M}_{1}+\dot{\varepsilon}_{\rho_{1}}-\frac{(\gamma+1) \dot{\varepsilon}_{\phi_{1}}-2 \dot{\varepsilon}_{\phi_{5}}}{\gamma-1} \\
& \dot{\chi}_{D F 5}=\dot{\rho}_{5}-\dot{\phi}_{5}-\frac{2 \gamma}{\gamma-1}\left(\dot{\phi}_{1}-\dot{\phi}_{5}\right)=\dot{M}_{5}+\dot{\varepsilon}_{\rho_{5}}-\frac{2 \gamma \dot{\varepsilon}_{\phi_{1}}-(\gamma+1) \dot{\varepsilon}_{\phi_{5}}}{\gamma-1}
\end{aligned}
$$

The symbols of $\rho$ and $\phi$ are pseudorange and carrier phase measurements, respectively. The subscripts 1 and 5 indicate L1 and L5 frequencies. The symbols of $M, \varepsilon$ and $\gamma$ are multipath, noise error and the squared ratio of L1 and L5 frequencies, respectively. The ENAC DF CCD monitors take the filtered rate of the DF measurement combinations by two cascades $1^{\text {st }}$ order smoothing filter with 30 -second of time constant. The threshold of this monitor is computed based on the statistics obtained from the Tenerife GBAS ground receiver, which will be given in the later section. The threshold of the ENAC DF CCD monitor has been determined according to the same approach used in previous research [7].

\section{ENAC Divergence-Free (DF) Innovation Monitor}

Since the existing Honeywell SQM and ENAC CCD monitors both have to some degree a delayed response time, in some rare fault cases within the threat space, they cannot achieve sufficiently low PMDs right after the fault onset. This can be problematic when the airborne filter is initialized at or right after the fault onset, which has been defined as the 'transient case'. To deal with this problem, this paper proposes the ENAC DF-Innovation Monitor, which has high sensitivity in detecting step-type errors. This monitor simply measures the difference between a predicted pseudorange and a raw pseudorange at current epoch as shown as the following equation.

$$
\begin{aligned}
Y_{k+1} & \equiv \hat{\rho}_{S C H E M E, k+1}-\rho_{S C H E M E, k+1} \\
& =\left[\tilde{\rho}_{S C H E M E, k}+\Delta \phi_{S C H E M E, k+1}\right]-\rho_{\text {SCHEME }, k+1}
\end{aligned}
$$

The subscripts $k$ and $k+1$ indicate previous and current epochs, respectively. In addition, the subscript, SCHEME can be either 'DF1' and 'DF5', which represent divergence-free (DF) L1 and L5 measurement combination. The operator tilde ( $\left(^{(}\right)$and $\Delta$ indicate the filtered value using $1^{\text {st }}$ order smoothing filter and a time difference operator. The computation of filtered $\rho_{S C H E M E}$ can be seen in Figure 2. And the $\rho_{\text {SCHEME }}$ and $\phi_{\text {SCHEME }}$ used for the DF1- and DF5-Innovation Monitors, filtered value of $\tilde{\rho}_{S C H E M E}$ and their error components are shown in Table 1. 


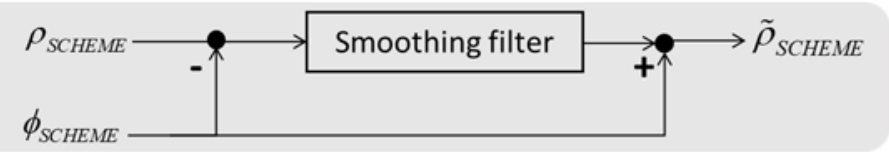

Figure 2 Block diagram for computation of $\tilde{\rho}_{\text {SCHEME }}$

Table 1 The $\rho_{S C H E M E}$ and $\phi_{S C H E M E}$ used for the DF1- and DF5-Innovation Monitors and their error components

\begin{tabular}{|c|c|c|c|c|}
\hline SCHEME & $\rho$ SCHEME & $\phi S C H E M E$ & $\tilde{\rho}_{\text {SCHEME }}$ & Error components in $Y$ \\
\hline DF1 & $\rho_{L 1}$ & $\phi_{1}+2 \frac{\phi_{1}-\phi_{5}}{\gamma-1}$ & $\begin{aligned} \tilde{\rho}_{D F 1}= & d-b+B+T+\tilde{M}_{1}+\tilde{\varepsilon}_{\rho 1} \\
& -\alpha_{1}\left(\tilde{\varepsilon}_{\phi 1}-\varepsilon_{\phi 1}\right)-\alpha_{2}\left(\tilde{\varepsilon}_{\phi 5}-\varepsilon_{\phi 5}\right)\end{aligned}$ & $\begin{array}{l}\left\{\tilde{M}_{\rho_{1}, k}-M_{\rho_{1}, k+1}\right\} \\
+\left\{\tilde{\varepsilon}_{\rho_{1}, k}-\varepsilon_{\rho_{1}, k+1}\right\}-\alpha_{1} \tilde{\varepsilon}_{\phi_{1}, k}-\alpha_{2} \tilde{\varepsilon}_{\phi_{3}, k}+\alpha_{1} \varepsilon_{\phi_{1}, k+1}+\alpha_{2} \varepsilon_{\phi_{5}, k+1}\end{array}$ \\
\hline DF5 & $\rho_{L 5}$ & $\phi_{5}+2 \gamma \frac{\phi_{1}-\phi_{5}}{\gamma-1}$ & $\begin{aligned} \tilde{\rho}_{D F 5} & =d-b+B+T+\tilde{M}_{5}+\tilde{\varepsilon}_{\rho 5} \\
& -\beta_{1}\left(\tilde{\varepsilon}_{\phi 1}-\varepsilon_{\phi 1}\right)-\beta_{2}\left(\tilde{\varepsilon}_{\phi 5}-\varepsilon_{\phi 5}\right)\end{aligned}$ & $\begin{array}{l}\left\{\tilde{M}_{\rho_{5}, k}-M_{\rho_{5}, k+1}\right\} \\
+\left\{\tilde{\varepsilon}_{\rho_{5}, k}-\varepsilon_{\rho_{5}, k+1}\right\}-\beta_{1} \tilde{\varepsilon}_{\phi_{1}, k}-\beta_{2} \tilde{\varepsilon}_{\phi_{5}, k}+\beta_{1} \varepsilon_{\phi_{1}, k+1}+\beta_{2} \varepsilon_{\phi_{5}, k+1}\end{array}$ \\
\hline Note & & & $\alpha_{1}=-\frac{\gamma+1}{\gamma-1}, \alpha_{2}=\frac{2}{\gamma-1}, \beta_{1}=$ & $\frac{2 \gamma}{-1}, \beta_{2}=\frac{\gamma+1}{\gamma-1}$ \\
\hline
\end{tabular}

To improve the performance of the monitor, the error level of the monitor needs to be reduced as much as possible. As shown in Table 1, the errors of the DF-Innovation monitor, denoted as $Y$, consist of multipath and noise errors. Looking closely at the equation, the multipath related term is the difference between the filtered multipath at the previous epoch and the raw multipath at the current epoch. Because the multipath is temporally correlated, this differenced value will have a smaller value if a small time constant for the smoothing filter is used. On the other hand, it is obvious that using a larger smoothing time constant is more effective in reducing error level of white noise components. That is, there is trade-off relationship in reducing error terms of multipath and noise error. To determine the time constant that is the most effective in reducing the error level of the DF-Innovation monitor, we collected two data sets from GBAS ground receivers in Barcelona and Tenerife Airports within the frame of the SESAR 2020 Pj14-03-01 WP8 T04. The former is equipped with choke ring antenna and the latter with Low-Noise Antenna (MLA). Both sites used Novaltel ProPak 6 receivers. The Barcelona data is collected on day-of-year (DOY) 316-317 in 2017 with $2 \mathrm{~Hz}$ sampling and the Tenerife data is collected on DOY 128 in 2019 with the same sampling rate. Using these data collections, the standard deviations of the DF1Innovation Monitors are computed according to elevation angles as shown in Figure 3. As a result, the smaller the time constant of the smoothing filter was used, the smaller the standard deviation of the monitor we could obtain. We think that the impact of multipath is dominant over that of noise error. In this paper, we choose to use a standard deviation obtained from Tenerife airport using the smoothing filter with 1-second time constant for the DF-Innovation Monitor when the measurement sampling interval is 0.5 second. Note that this is not the final decision of the time constant for the proposed monitor. More data analysis is needed to finalize the design of the DF-Innovation Monitor. But for now, a 1-second time constant is used for filtering the DF-Innovation Monitor. The obtained standard deviations for the ENAC CCD and DF-Innovation monitors are summarized in Table 2. We took standard deviation computed for low elevation angles (5-10deg) for conservative analysis.

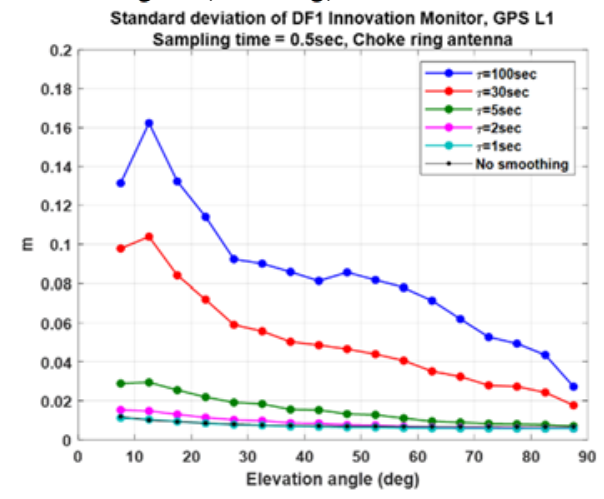

(a)

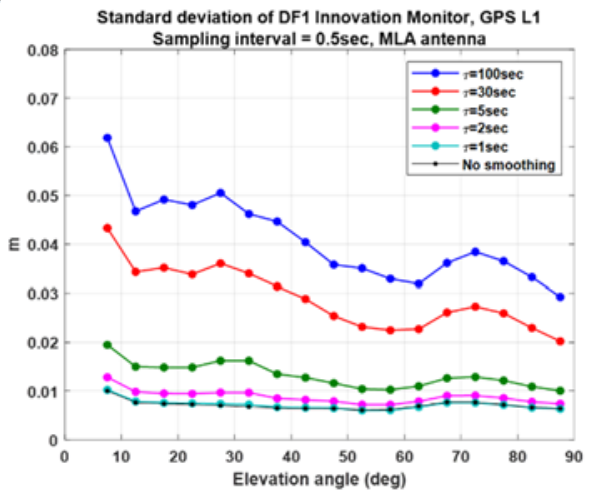

(b)

Figure 3 Standard deviation of DF1-Innovation Monitor at (a) Barcelona airport (Choke-ring antenna) and (b) Tenerife airport (MLA antenna) 
Table 2 The standard deviation obtained from Tenerife GBAS ground receiver for ENAC CCD and DF-Innovation monitors

\begin{tabular}{|c|c|c|c|c|}
\hline & \multicolumn{2}{|c|}{ GPS } & \multicolumn{2}{c|}{ Galileo } \\
\hline Monitors & Standard deviation & Over-bound factor & Standard deviation & Over-bound factor \\
\hline ENAC CCD DF1 & $0.0009 \mathrm{~m} / \mathrm{s}$ & 2.53 & $0.0028 \mathrm{~m} / \mathrm{s}$ & 1.39 \\
\hline ENAC CCD DF5 & $0.0011 \mathrm{~m} / \mathrm{s}$ & 1.76 & $0.0028 \mathrm{~m} / \mathrm{s}$ & 1.82 \\
\hline $\begin{array}{c}\text { DF1-Innovation } \\
\text { Monitor }\end{array}$ & $0.0102 \mathrm{~m}$ & 1.29 & $0.0086 \mathrm{~m}$ & 1.41 \\
\hline $\begin{array}{c}\text { DF5-Innovation } \\
\text { Monitor }\end{array}$ & $0.0113 \mathrm{~m}$ & 1.20 & $0.0124 \mathrm{~m}$ & 1.37 \\
\hline
\end{tabular}

The over-bound factor is determined such that the inflated theoretical normal distribution bounds the actual distribution based on a Q-Q plot. The threshold of the DF-Innovation monitor is determined based on the continuity requirement of signal deformation fault given as $1.5 \times 10^{-7}$ per 15 seconds. When we compute the probability of false alarm rate, we assume the worst case that there are 15 independent samples during the 15 -second of exposure time and 15 satellites being tracked simultaneously.

\section{REQUIREMENT ON RANGE FAULT MONITOR FOR GAST D}

The requirement on the ranging source fault monitor for GAST D has been defined in the standard document as shown in Figure 4 [2]. For GAST F, this requirement may be relaxed in the future since the satellite geometry will be improved for the multiconstellation geometries. However, this requirement specific for GAST F has not been defined yet in the standards document, so the same requirement for GAST D is used for GAST F in this assessment. For the assessment of the performance of the signal quality monitors, the differential range error and the PMD needs to be computed correctly. As recommended in the standard document [2], ground latency, which represents the correction broadcast delay and margin, is considered when computing differential range error and integrity message latency and time-to-alert is taken into account when computing the PMD. The values for these parameters will be given in the next section.

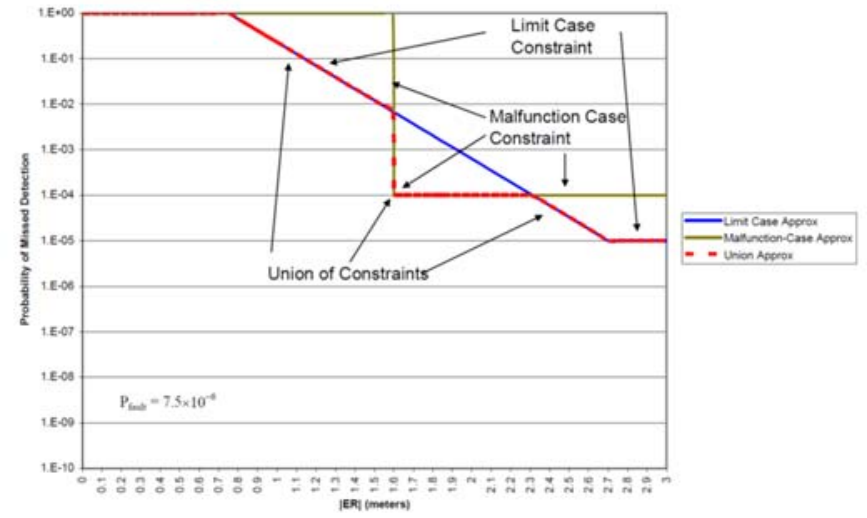

Figure 4 PMD constraints according to differential range error [2]

\section{SIMULATION ENVIRONMENT}

In this study, the PMD compliance of the monitors for the satellite signal deformation is assessed in two modes. One is for singlefrequency mode and the other is for dual-frequency mode. For single-frequency mode, the performance of the signal distortion monitors is assessed on GPS L1, Galileo E1c and GPS L5/Galileo E5a separately, assuming that the GAST D can be applied not only for GPS L1 but also for other GNSS signals on single-frequency basis. For dual-frequency mode, the test assumes that a single fault occurs on one frequency. The performance of the monitors is assessed for the following types of test.

- GPS L1/L5: a single fault occurred on L1 frequency, DF1 monitors are used under ionosphere-free (IF) navigation mode

- GPS L1/L5: a single fault occurred on L5 frequency, DF5 monitors are used under IF navigation mode

- Galileo E1/E5: a single fault occurred on E1c frequency, DF1 monitors are used under IF navigation mode

- Galileo E1/E5: a single fault occurred on E5a frequency, DF5 monitors are used under IF navigation mode 


\section{Assessment Process}

Simulations have been performed to investigate the monitor performance and to check under what conditions compliance with the requirement is achieved. The simulation has the following procedure: firstly, the GNSS code sequences are generated and the distortion is added onto the code sequences according to values given by the threat model parameters, which represent the lead/lag of a chip, resonant frequency and damping factor. This is made for the full range of parameter combinations within the threat space. Secondly, the correlation function is computed, and it is applied to the pre-correlation filters according to the types of filters and associated bandwidths. By inspecting the filtered correlation function with the Early-Late correlator, the code tracking offset is computed. The resultant code delay can vary according to the values of chip spacings selected for Early-Late correlator. Thirdly, the range error can be computed by comparing code delay values from nominal and distorted correlation functions, and it represents the error associated with the signal deformation. The ground and airborne receivers experience different range errors if they have different receiver configurations. Finally, the computed range error is added to the simulated pseudorange measurement. This procedure has been programmed in Matlab by ENAC [5] and validated for several years.

\section{Receiver configuration}

For some specific satellite signal deformations, the effect on the range error can differ according to receiver configurations in terms of precorrelation bandwidth, Early-Late correlator chip spacing and the types of precorrelation filter used. To take into account all possible cases of differential range errors, the test has been conducted for various receiver configurations at the airborne receiver. Considering the GPS tracking constraints given in [12], the precorrelation bandwidth and Early-Late correlator chip spacing for ground airborne receivers are set as shown in Figure 5. It should be noted that there are no tracking constraints defined for GPS L5, Galileo E1c/E5a signals yet. For those signals, the types of precorrelation filters are set to the same ones used by [5]. At the ground receiver, 6th-order Butterworth filter was used and at airborne receiver, the following 4 types of filters are tested: 6th-order Butterworth filter, 0-group delay resonator, $150 \mathrm{~ns}$ differential group delay resonator and $150 \mathrm{~ns}$ differential group delay resonator.
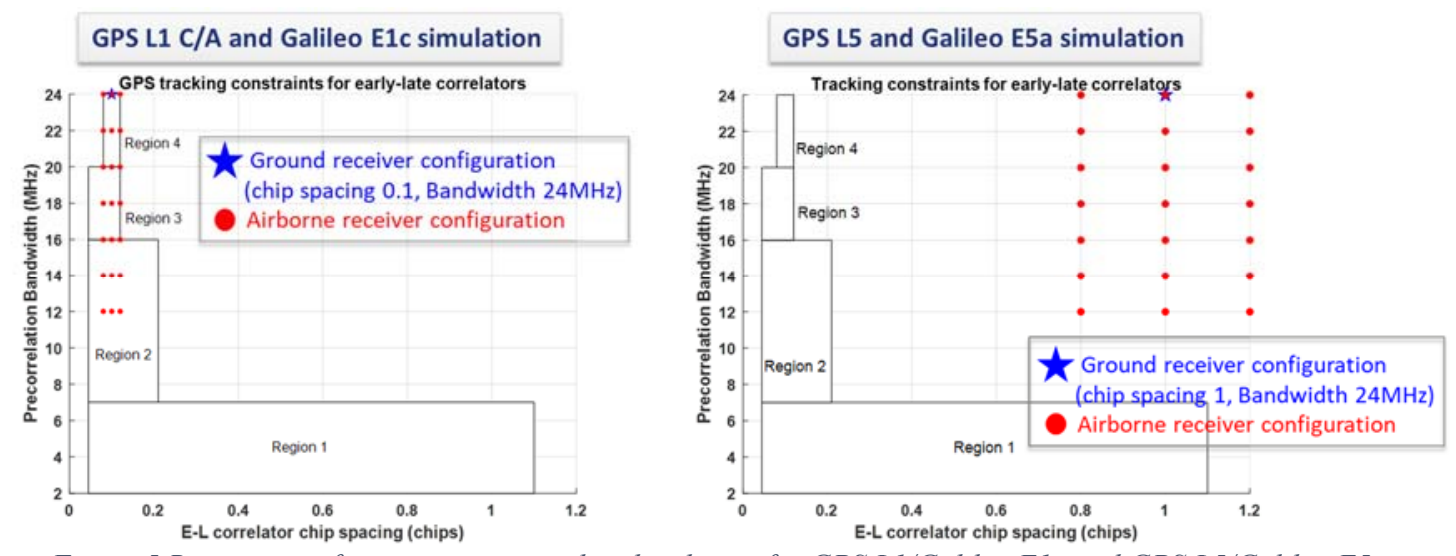

Figure 5 Receiver configuration at ground and airborne for GPS L1/Galileo E1c and GPS L5/Galileo E5a

\section{Threat spaces for GPS L1/L5 and Galileo E1c/E5a signals}

The signal deformation fault for GPS L1 C/A has been defined in the standard document [3]. There are three types of threat models, which are classified into threat model A, B and C. Threat model A represents the digital fault when the chips are leaded or lagged to some amount of chips $(\Delta)$. Threat model B is the analog faults, which adopts the unit step response of a second order system that is modeled by resonant frequency $\left(\mathrm{f}_{\mathrm{d}}\right)$ and damping factor $(\sigma)$. There has not been defined standard model for GPS L5 and Galileo E1c/E5a signals yet, however, [5] proposed the threat models for those signals using the same threat model structures. This paper adopts the threat model in [5] for GPS L5 and Galileo E1c/E5a signals. Table 3 and Table 4 show the threat model parameters for all signals.

Table 3 Threat model parameter of GPS L1 C/A [3]

\begin{tabular}{|l|l|l|l|}
\hline & \multicolumn{1}{|c|}{ TMA } & \multicolumn{1}{|c|}{ TMB } & \multicolumn{1}{c|}{ TMC } \\
\hline & $\Delta=-0.12: 0.01: 0.12$ (chip) & $\Delta=0$ (chip) & $\Delta=-0.12: 0.01: 0.12($ chip) \\
GPS L1 & $\mathrm{f}_{\mathrm{d}}=0(\mathrm{MHz})$ & $\mathrm{f}_{\mathrm{d}}=4: 0.1: 17(\mathrm{MHz})$ & $\mathrm{f}_{\mathrm{d}}=7.3: 0.1: 13(\mathrm{MHz})$ \\
& $\sigma=0$ (Mnepers/s) & $\sigma=0.8: 0.5: 8.8(\mathrm{Mnepers} / \mathrm{s})$ & $\sigma=0.8: 0.5: 8.8(\mathrm{Mnepers} / \mathrm{s})$ \\
\hline
\end{tabular}




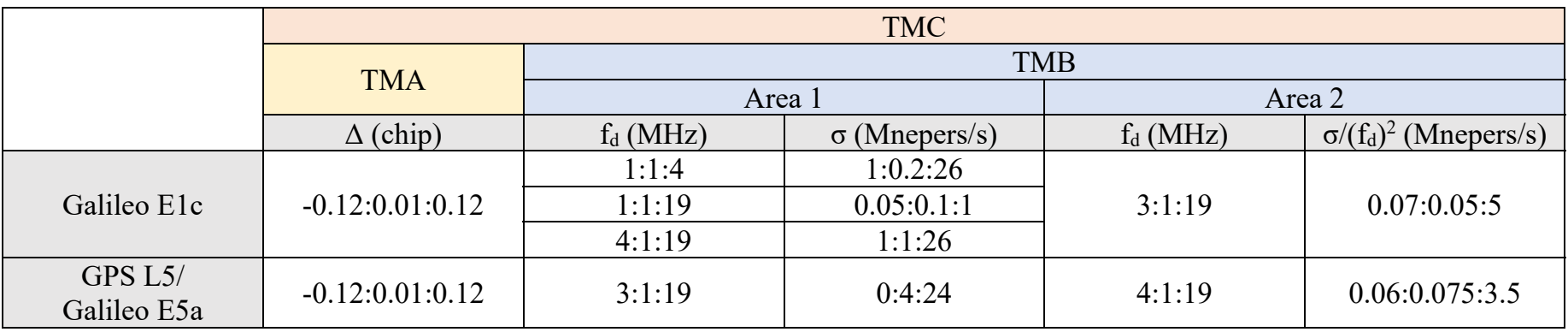

\section{Test configuration}

The timing values that are used in this test are defined in Table 5. The latency of the correction message is set to different values for GAST D and F because the message update interval will be increased in GAST F $[8,10]$. On the other hand, the update interval of the integrity message will stay the same for GAST F, the value of 1.5 second is set for GAST D and F. Time-To-Detect and Affect the Broadcast (TTDABA) is 1.5 seconds for both GAST D and F, because the time-to-alert requirement will not change for GAST F $[2,8]$. The time constant used for smoothing filter is set to 30second for GAST D and 100second for GAST F [7, 10]. The fault onset time is set to 180 second, and the ground filter is assumed to be initialized at 0 second.

Table 5 Test configuration

\begin{tabular}{|c|c|}
\hline Simulation parameters & Values \\
\hline Latency of correction message $\left(\tau_{\mathrm{G}}\right)$ & $\begin{array}{l}\text { GAST D: } 1.5 \mathrm{sec} \text { (message update interval } 0.5 \mathrm{sec}+\text { ground delay } 1 \mathrm{sec}) \\
\text { GAST F: } 3 \mathrm{sec} \text { (message update interval } 2.0 \mathrm{sec}+\text { ground delay } 1 \mathrm{sec})[8,10]\end{array}$ \\
\hline Latency of Integrity message $\left(\tau_{\mathrm{I}}\right)$ & $\begin{array}{l}\text { GAST D: } 1.5 \mathrm{sec} \text { (message update interval } 0.5 \mathrm{sec}+\text { ground delay } 1 \mathrm{sec}) \\
\text { GAST F: } 1.5 \mathrm{sec} \text { (message update interval } 0.5 \mathrm{sec}+\text { ground delay } 1 \mathrm{sec})[8]\end{array}$ \\
\hline $\begin{array}{l}\text { Time-To-Detect and Affect the Broadcast } \\
\text { (TTDABA) }\end{array}$ & $\begin{array}{l}\text { GAST D, GAST F: } 1.5 \mathrm{sec}(0.5 \mathrm{sec} \text { missed integrity messages }+1.0 \mathrm{sec} \text { margin }) \\
{[2,8]}\end{array}$ \\
\hline Time constant for carrier smoothing & $\begin{array}{l}\text { GAST D: } 30 \mathrm{sec} \\
\text { GAST F: } 100 \mathrm{sec}\end{array}$ \\
\hline Time of fault onset $\left(\mathrm{t}_{\mathrm{f}}\right)$ & $180 \mathrm{sec}$ \\
\hline Filter initialization time at ground $\left(\mathrm{t}_{\mathrm{g}}\right)$ & $0 \mathrm{sec}$ \\
\hline Filter initialization time at airborne $\left(\mathrm{t}_{\mathrm{a}}\right)$ & $\begin{array}{l}\text { Post-convergence case: } 50 \mathrm{sec} \\
\text { Transient case: } 180 \mathrm{sec}\end{array}$ \\
\hline
\end{tabular}

We considered two cases for the airborne filter initialization time: one represents the post-convergence case and the other is transientcase. These two cases need to be taken into account because there is a possibility of having various differential range error even under the same fault cases and receiver configurations. Figure 6 shows time histories of the range error at ground and airborne, associated differential range error and ground monitor for post-convergence and transient cases. The post-convergence case indicates when the airborne filter is initialized far earlier than the time of the fault onset. In this case, the differential range error gradually increases starting from zero and at the same time, the ground monitor metric also gradually increases after the fault onset. If you recall the PMD requirement defined in Figure 4, there is a high possibility that the associated situation can be plotted within the requirement PMD, that is, the PMD compliance region. On the other hand, in the transient-case, the airborne filter is initialized at or right after the fault onset, and accordingly, the differential range error starts from nonzero value and gradually decreases. However, as the ground monitor behaves as the same as the post-convergence case, there is a high change that the associated differential range error and the PMD of ground monitor falls outside of the PMD requirement plot. Therefore, it may be more difficult to achieve compliance for the monitors in the transient case. To deal with this problem, the term 'time delay', denoted as $t_{\text {delay }}$, is introduced, which indicates the time from the airborne filter initialization to the moment when the airborne incorporates the correction and measurement for navigation. Generally, the value of 50 seconds is recommended for this parameter [7]. 


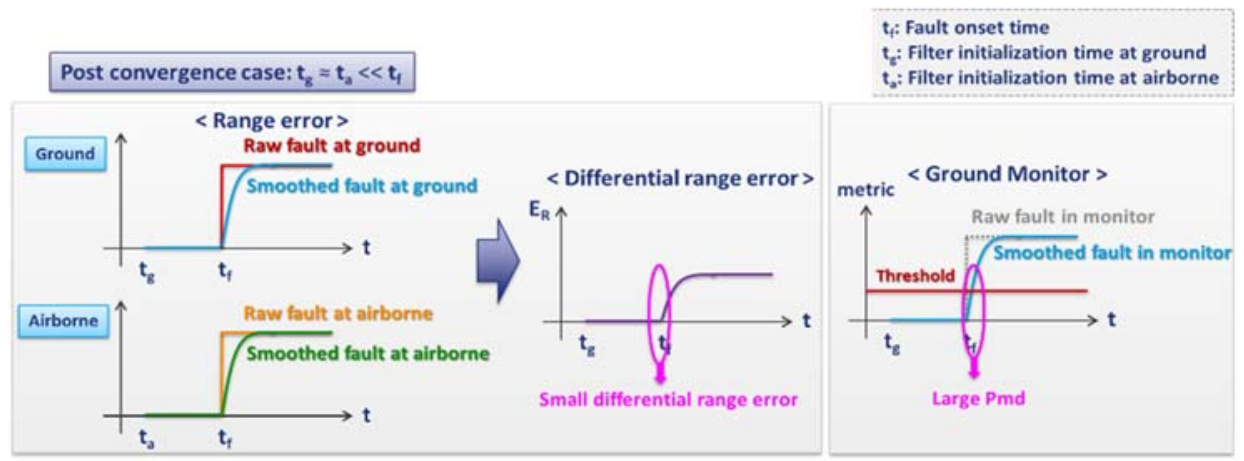

(a)
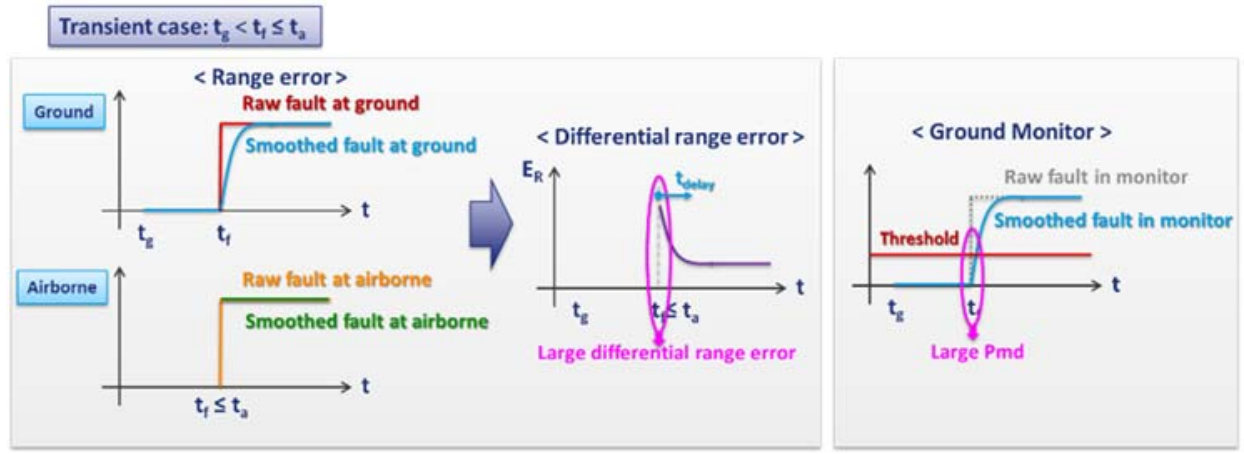

(b)

Figure 6 Differential range error and ground monitor for (a) post-convergence case and (b) transient case

\section{TEST RESULTS}

Firstly, the performance of the monitors for single frequency mode is assessed. Table 6 shows the PMD compliance results for all fault cases and receiver configurations. The green shade indicates the compliance and the red shade indicates the non-compliance. During the assessment, the time delay, $t_{\text {delay }}$ is set to 50 seconds for transient case, which is commonly used value for this parameter. For GPS L1 signal, the time constant for carrier smoothing filter, TTDABA and ground latency are recommended in the standard document. However, those values for other signals are not recommended yet, therefore two values of time constant for carrier smoothing filter is tested for GPS L5 and Galileo E1c/E5a signals. In the meanwhile, the update interval of the correction message is planned to increase for multi-constellation and multi-frequency modes, the latency of correction message is set to 3.0 seconds for GPS L5 and Galileo E1c/E5a signals. As a result, the PMD of the monitors was compliant for all fault cases and receiver configurations for GPS L1 signal. However, for Galileo E1c signal, the PMD was not compliant for the TMB and TMC fault cases under post-convergence case when 30-second of time constant is used for a carrier smoothing filter. If the time constant is increased to 100 seconds, the PMD was compliant for all fault cases under post-convergence case, however, there was one non-compliant result only for the TMA-type fault under transient case. For GPS L5 or Galileo E5a signal, the PMD was all compliant when 30-second of time constant for smoothing filter is used. If the time constant is increased to 100 seconds, for transient case, the PMD becomes noncompliant. For transient case, to make the monitor become compliant with the requirement, $t_{\text {delay }}$ needs to be increased over 50 seconds.

Table 6 PMD compliance results for single-frequency mode (GAST D, GAST D like)

\begin{tabular}{|c|c|c|c|c|c|c|}
\hline & & \multicolumn{4}{c|}{ PMD compliance results } \\
\hline & & GPS L1 & \multicolumn{2}{c|}{ Galileo E1c } & \multicolumn{2}{c|}{ GPS L5/Galileo E5a } \\
\hline \multicolumn{2}{|c|}{$\begin{array}{c}\text { Time constant for } \\
\text { carrier smoothing }\end{array}$} & $30 \mathrm{sec}$ & $30 \mathrm{sec}$ & $100 \mathrm{sec}$ & $30 \mathrm{sec}$ & $100 \mathrm{sec}$ \\
\hline \multirow{2}{*}{ TMA } & $\begin{array}{c}\text { Post- } \\
\text { convergence }\end{array}$ & & & & & \\
\cline { 2 - 6 } & Transient & & & & & \\
\hline
\end{tabular}




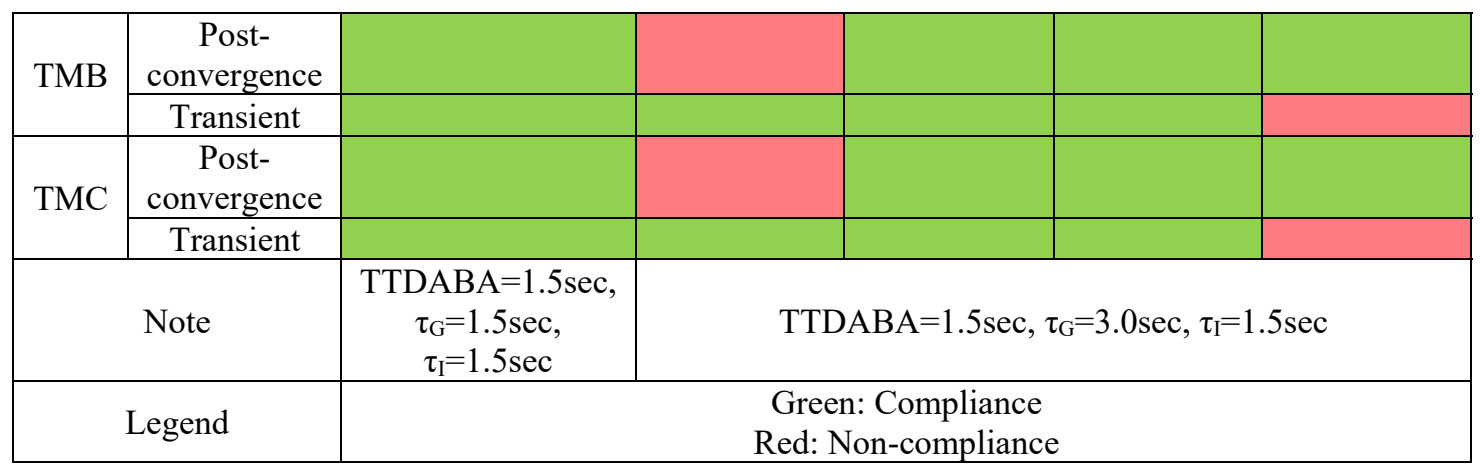

Secondly, the performance of the monitors for dual-frequency modes is assessed. Table 7 shows that the PMD of the monitors was compliant under all fault cases and all receiver configurations for all signals when Honeywell SQM, ENAC CCD and ENAC DFInnovation monitors are used. For transient case, the value of $t_{\text {delay }}$ is set to 50 seconds as the previous test. We think that the reason why the PMD was compliant for all cases for dual-frequency mode unlike single-frequency mode is because the performance of the ENAC CCD monitor has been improved through elimination of the ionospheric delay in the monitor and the use of the proposed ENAC DF-Innovation monitor.

Table 7 The PMD compliance results for dual-frequency mode (GAST F) when Honeywell SQM, ENAC CCD and ENAC DF-Innovation monitors are used

\begin{tabular}{|c|c|c|c|c|c|}
\hline & & \multicolumn{4}{|c|}{ PMD compliance results } \\
\hline & & GPS L1 & Galileo E1c & GPS L5 & Galileo E5a \\
\hline \multicolumn{2}{|c|}{$\begin{array}{l}\text { Time constant for } \\
\text { carrier smoothing }\end{array}$} & \multicolumn{4}{|c|}{$100 \mathrm{sec}$} \\
\hline \multirow[t]{2}{*}{ TMA } & $\begin{array}{c}\text { Post- } \\
\text { convergence }\end{array}$ & & & & \\
\hline & Transient & & & & \\
\hline \multirow[t]{2}{*}{ TMB } & $\begin{array}{c}\text { Post- } \\
\text { convergence }\end{array}$ & & & & \\
\hline & Transient & & & & \\
\hline \multirow[t]{2}{*}{ TMC } & $\begin{array}{c}\text { Post- } \\
\text { convergence }\end{array}$ & & & & \\
\hline & Transient & & & & \\
\hline & Note & \multicolumn{4}{|c|}{$\begin{array}{l}\text { Green: Compliance } \\
\text { Red: Non-compliance }\end{array}$} \\
\hline
\end{tabular}

To see the advantage of using ENAC DF-Innovation monitor, the required value of $t_{\text {delay }}$ is plotted for Galileo E1c transient case under TMC2 fault cases when the value of $\Delta$ equals to -0.02 chip in Figure 7. The left figure is plotted for the case when only Honeywell SQM and ENAC CCD monitors are used, and the right figure shows the result when the ENAC DF-Innovation monitor is additionally used. For some specific values of $f_{d}$ and $\sigma / f_{d}{ }^{2}$, the first case requires at least 145 seconds of $t_{\text {delay }}$ to make the monitor become compliant with the requirement. On the other hand, when the ENAC DF-Innovation monitor is added, the required $t_{\text {delay }}$ is reduced to 30 seconds where the gain is approximately $80 \%$. Table 8 summarizes the required $t_{\text {delay }}$ for each signal and for two cases where the one without the ENAC DF-Innovation monitor and the other with the proposed monitor. For the monitors to be compliant with the requirement regardless of types of signals, the first case needs minimum 145 seconds of $t_{\text {delay }}$, on the other hand, the second case with the proposed monitor requires only 30 seconds. To sum up, the addition of the ENAC DF-Innovation monitor can reduce the required $t_{\text {delay }}$ by 80 and thus avoid unnecessary delays for the use of newly available satellites. Moreover, it also suggests that the addition of the ENAC DF-Innovation monitor can even reduce the recommended $t_{\text {delay }}$ from 50 seconds to 30 seconds if considering only the signal deformation fault. 


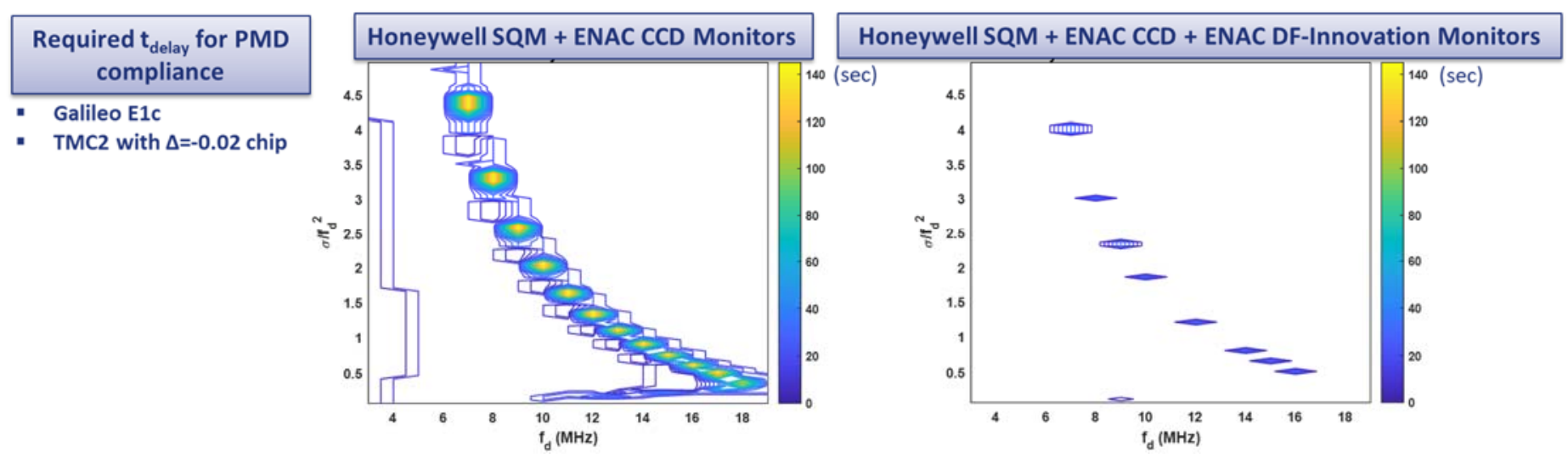

Figure 7 Required tdelay for (Honeywell SQM+ENAC CCD) vs. (Honeywell SQM+ENAC CCD+ENAC DF-Innovation monitor) for Galileo E1c, TMC2 case

Table 8 Required tdelay for all signals and its overall value for (Honeywell $S Q M+E N A C C C D)$ vs. (Honeywell $S Q M+E N A C C C D+E N A C D F-$ Innovation monitor)

\begin{tabular}{|c|c|c|c|c||c|}
\hline & \multicolumn{5}{|c|}{ Minimum required $t_{\text {delay }}(\mathrm{sec})$} \\
\hline & GPS L1 & GAL E1c & GPS L5 & GAL E5a & Overall \\
\hline $\begin{array}{c}\text { Honeywell SQM } \\
\text { +ENAC CCD }\end{array}$ & 30 & 145 & 20 & 20 & $\mathbf{1 4 5}$ \\
\hline $\begin{array}{c}\text { Honeywell SQM } \\
\text { +ENAC CCD } \\
\text { +ENAC DF-Innovation Monitor }\end{array}$ & 30 & 30 & 20 & 20 & $\mathbf{3 0}$ \\
\hline
\end{tabular}

\section{CONCLUSIONS}

This paper proposed the ENAC DF-Innovation monitor, which can improve detection performance right after the fault onset where the existing Honeywell SQM and ENAC CCD monitors are vulnerable due to the response time of each metric. By analyzing the error components in the DF-Innovation monitor, a 1-second time constant was determined which can reduce the errors in the monitor to the lowest level for two data collections at Barcelona and Tenerife airports. Since not every detail has been standardized for GAST F so far, the values of a time constant for carrier smoothing filter, latencies of correction and integrity messages and TTDABA are selected appropriately based on the recent agreements on GAST F within SESAR studies and former research. In addition, the threat spaces for GPS L5 and Galileo E1c/E5a are adopted from the previous ENAC work.

The assessment has been conducted in two modes: single-frequency mode (GAST D or GAST D like) and dual-frequency mode (GAST F). In the results for the single-frequency mode, the PMD was compliant for GPS L1 signal for all fault cases and receiver configurations. For Galileo E1c, the PMD was compliant except for the TMB and TMC under post-convergence case when the 30 seconds of time constant was used, and for the TMA under transient case when the time constant was set to 100 seconds. For Galileo E5a, all cases are compliant when a 30-second time constant for the carrier smoothing filter is used. However, if the time constant is increased to 100 seconds, all transient cases become non-compliant. Unlike the single-frequency mode, the PMD was compliant with the requirement for all fault cases and receiver configurations for all signals under the dual-frequency mode. The improvement of the performance of the ENAC CCD monitor due to the elimination of the ionospheric delay and the utilization of the ENAC DFInnovation monitor seem to play a critical role for making PMD compliant for dual-frequency mode. We also check the advantage of using the proposed ENAC DF-Innovation monitor that it can reduce the required $t_{\text {delay }}$ by $80 \%$ compared to the case without using the proposed monitor. The proposed monitor also can reduce the recommended $t_{\text {delay }}$ below 50 seconds, which can allow airborne users to wait less time to compute their positions.

In this research, only a single fault on a single frequency has been considered. We need to check if there is any possibility of having dual faults on two frequencies simultaneously. However, the general consensus appears to be that this threat may be neglected [13]. In addition, the minimum PMD among the PMDs of three monitors is used for assessment of the PMD compliance in this test. We 
think that it is more realistic to compute the PMD by considering the correlations among monitors in the future. Furthermore, we are planning to use the extended threat space for Galileo signals which are discussed upon for standardization for the PMD assessment when it becomes available.

\section{ACKNOWLEDGEMENTS}

This work is funded by Single European Sky ATM Research (SESAR) Joint Undertaking within the frame of the SESAR 2020 Pj1403-01 WP8 T04. The authors would like to thank INDRA Navia, INDRA Satnav and ENAIRE for the use of data collection under the SESAR Program.

\section{REFERENCES}

1. Daly, P., Riley, S., Raby, P., "Recent Advances in the Implementation of GNSS," Proceedings of the 6th International Technical Meeting of the Satellite Division of The Institute of Navigation (ION GPS 1993), Salt Lake City, UT, September 1993, pp. 433-440.

2. "Conceptual Framework for the Proposal for GBAS to Support CAT III Operations", NSP WGW November 2009 Report

3. Brenner, M., Liu, F. "Ranging Source Fault Detection Performance for Category III GBAS", 23rd International Technical Meeting of the Satellite Division of The Institute of Navigation, Portland, OR, September 21-24, 2010, pp.2618-2632.

4. ICAO, “Aeronautical Telecommunications Volume 1 Radio Navigation Aids", Annex 10, 2018.

5. Pagot, J.-B. "Modeling and Monitoring of New GNSS Signal Distortions in the Context of Civil Aviation", PhD Dissertation, Ecole Nationale de l'Aviation Civile, France, 2016.

6. Liu, F., Brenner, M., Tang, C.Y., "Signal Deformation Monitoring Scheme Implemented in a Prototype Local Area Augmentation System Ground Installation," Proceedings of the 19th International Technical Meeting of the Satellite Division of The Institute of Navigation (ION GNSS 2006), Fort Worth, TX, September 2006, pp. 367-380.

7. Jiang, Y., Milner, C. and Macabiau, C. "Code carrier divergence monitoring for dual-frequency GBAS", GPS Solutions, Springer Verlag, 2017, 21 (2), pp. Pages 769-781.

8. Milner, C., Guilbert, A., Macabiau, C. "Evolution of Corrections Processing for the MC/MF Ground Based Augmentation System (GBAS)," Proceedings of the 2015 International Technical Meeting of The Institute of Navigation, Dana Point, California, January 2015, pp. 364-373.

9. Brenner, M., Liu, F., "Ranging Source Fault Detection Performance for Category III GBAS," Proceedings of the 23rd International Technical Meeting of the Satellite Division of The Institute of Navigation (ION GNSS 2010), Portland, OR, September 2010, pp. 2618-2632.

10. SESAR, D3.7.1 Monitoring, Integrity and Performance, 03.00.00, November 2015.

11. Pervan, Boris, Khanafseh, Samer, Patel, Jaymin, "Test Statistic Auto- and Cross-correlation Effects on Monitor False Alert and Missed Detection Probabilities," Proceedings of the 2017 International Technical Meeting of The Institute of Navigation, Monterey, California, January 2017, pp. 562-590.

12. Minimum Operational Performance Standards (MOPS) for GPS Local Area Augmentation System Airborne Equipment, RTCA DO-253C, 2008.

13. "Baseline development standards for the validation of draft Annex 10, Volume I amendments relating to dual-frequency, multi-constellation (DFMC) SBAS", Appendix A to the Report on Agenda Item 2 of the Fifth Meeting of the Navigation Systems Panel (NSP/5). 